\title{
Antiferromagnetic Structure and Electronic Properties of $\mathrm{Mn}_{2} \mathrm{PtSn}$
}

\author{
G. Elouered ${ }^{a}$, D. Bensaid ${ }^{b, c, *}$, F. Benzoudji ${ }^{a}$, O. Arbouche $^{d}$, Z. Bouyakoub ${ }^{b}$, \\ N. MOUlay ${ }^{b}$ AND M. Ameri ${ }^{a}$ \\ ${ }^{a}$ Physics Department, Faculty of Science, University of Sidi-Bel-Abbes, 22000, Algeria \\ ${ }^{b}$ Laboratory Physico-Chemistry of Advanced Materials,University of Djillali Liabes, \\ BP 89, Sidi-Bel-Abbes, 22000, Algeria \\ ${ }^{c}$ Institute of Science, University Belhadj Bouchaib, BP 284, Ain-Temouchent, 46000, Algeria \\ ${ }^{d}$ DrTahar Moulay University of Saida, 20000 Saida, Algeria
}

(Received April 8, 2018; May 1, 2019; in final version May 3, 2019)

\begin{abstract}
$\mathrm{Mn}_{2} \mathrm{PtSn}$ tetragonal is promising for spintronic devices, especially for spin-transfer-torque based devices. We apply the density functional theory formalism to make a detailed study of the structural, magnetic, and electronic properties for $\mathrm{Mn}_{2} \mathrm{PtSn}$ crystals. The dependence of the elastic constants $C_{i j}$ for cubic and tetragonal structure, the aggregate elastic moduli $B, G$, and the anisotropy $A$, along with the related mechanical properties are investigated. The Curie temperatures and the magnetic moments in both the cubic as well as tetragonal phases have been calculated. $\mathrm{Mn}_{2} \mathrm{PtSn}$ can be studied in inverse tetragonal crystal structure with antiferromagnetic spin order. Because of the high magnetic anisotropy, low magnetization, and high spin polarization at the Fermi level, this material is expected to be promising for spin-transfer-torque applications.
\end{abstract}

DOI: 10.12693/APhysPolA.136.42

PACS/topics: $\mathrm{Mn}_{2}$-based Heusler alloys, magnetic properties, first-principles calculations, spin-polarized electronic bands

\section{Introduction}

The groups of Mn-based tetragonal compounds from the Heusler family are very important materials and have attracted considerable attention for their potential applications in many areas. In this family of compounds, $\mathrm{Mn}_{2} \mathrm{PtSn}$ has attracted considerable attention due to its hard-magnetic behavior and possible applications in spintransfer-torque (STT) based devices [1-3].

$\mathrm{Mn}_{2} \mathrm{PtSn}$ has been predicted to have ferromagnetic spin phase at the Curie temperature $T_{\mathrm{C}}=374 \mathrm{~K}$ in the inverse-tetragonal crystal structure (space group No. $119, I \overline{4} m 2)$ with high magnetocrystalline anisotropy $\left(\sim 50 \mathrm{Merg} / \mathrm{cm}^{3}\right)$ and high spin polarization $(P=91 \%)$ at the Fermi level $[4,5]$.

$\mathrm{Mn}_{3} \mathrm{Sn}$ has been predicted to crystallize in both hexagonal $D 0_{19}$ and tetragonal $D 0_{22}$ crystal structures, but only the hexagonal phase has been characterized in experiment [6]. Nelson et al. [7] have studied $\mathrm{Mn}_{3-x} \mathrm{Pt}_{x} \mathrm{Sn}$ $(x=0,0.5,1)$ nanomaterials, and confirmed that the $\mathrm{Mn}_{2} \mathrm{PtSn}$ has mainly inverse tetragonal structure with the ferro- (or ferrimagnetic) behavior at room temperature and has carried relatively low magnetization, high magnetic anisotropy, and low Curie temperature.

$\mathrm{Mn}_{2} \mathrm{PtSn}$ has been predicted to crystallize in two crystalline structures, cubic $F \overline{4} 3 m$ and inverse tetragonal $I \overline{4} m 2$ crystal structures. However, only the inverse

* corresponding author; e-mail:

djillali.bensaid@cuniv-aintemouchent.dz tetragonal phase $(a=4.512 \AA, c=6.084 \AA)$ has been synthesized in experiment by X-ray diffraction (XRD) [7].

Thus, in the present work, we investigate the elastic and the electronic structure properties of $\mathrm{Mn}_{2} \mathrm{PtSn}$ by using the full-potential linearized augmented plane wave. In Sect. 2, we have made a brief review of the theoretical method. The results and some discussions are presented in Sect. 3. Finally, in Sect. 4 we summarize conclusions drawn from our study.

\section{Theory method}

First principle calculations, based on the density functional theory (DFT), have shown a good accuracy in the study of many physical and chemical properties for a wide scale of materials. The full-potential linearized augmented plane wave (FP-LAPW) method, used for these calculations [8], was implemented in Wien2k code [911]. The exchange-correlation (XC) potentials were considered by the Perdew-Burke-Ernzerhof parameterization of the generalized gradient approximations (GGAPBE) $[12,13]$. The muffin tin radii (RMT) were chosen to ensure nearly touching spheres and minimizing the interstitial space. $\mathrm{RMT} \times K_{\max }=7$ (where $K_{\max }$ is the magnitude of the largest $K$ vector in the plane wave and RMT is the average radius of the muffin tin spheres) was used for the number of plane waves, and the expansion of the wave functions was set to $l_{\max }=10$ inside of the muffin tin spheres, while the charge density was Fourier expanded up to $G_{\max }=12$ (a.u. $)^{-1}$, where $G_{\max }$ is the parameter, which, in charge density Fourier expansion, sets the magnitude of largest vector to 14 . The MonkhorstPack method in the Brillouin zone for our compounds 
is performed with 2000 special $k$-points [14]. To set up the separation of valence and core states, the cut-off energy was chosen as -7 Ry. During the self-consistency cycles we select the total energy convergence as $10^{-5} \mathrm{Ry}$.

\section{Results and discussion}

\subsection{Structural properties}

$\mathrm{Mn}_{2} \mathrm{PtSn}$ crystallizes into both structures. The first, in non-centrosymmetric $I \overline{4} m 2$ structure with two nonequivalent Wyckoff positions occupied by $\mathrm{Mn}$ atoms: $\mathrm{Mn}_{1}$ at $2 \mathrm{~b}(0,0.5,0)$ and $\mathrm{Mn}_{2}$ at $2 \mathrm{~d}(0,0.5,0.75)$. $\mathrm{Pt}$ and Sn elements occupy the $2 \mathrm{a}(0,0,0)$ and $2 \mathrm{c}(0$, $0.5,0.25)$ positions. Secondly, the cubic structure with $\mathrm{Hg}_{2} \mathrm{CuTi}$ type exhibits $\mathrm{Td}$ symmetry (space group no $216, F \overline{4} 3 m$ ). In that structure the $\mathrm{Mn}_{2}$ atoms occupy the non-equivalent $4 \mathrm{~b}, 4 \mathrm{~d}$ Wyckoff positions at $(0.5,0.5,0.5)$ and $(0.75,0.75,0.75)$. The $\mathrm{Pt}$ and $\mathrm{Sn}$ are located on $4 \mathrm{c}$ $(0.25,0.25,0.25)$ and $4 \mathrm{a}(0.0,0.0,0.0)$ positions, respectively, as shown in Fig. 1 with a schematic overview of the type of magnetic order i.e. the Mn moments are oriented perpendicular to the respective planes but the moments have slightly different magnitudes in the two planes. The $\mathrm{Mn}_{1}$ moments in the $\mathrm{Mn}-\mathrm{Sn}$ plane are coupled antiferromagnetically to the $\mathrm{Mn}_{2}$ moments in the $\mathrm{Mn}-\mathrm{Pt}$ plane. The valence electrons of $\mathrm{Mn}, \mathrm{Pt}$ and $\mathrm{Sn}$ are $7\left(3 d^{5} 4 s^{2}\right), 10$ $\left(5 d^{9} 6 s^{1}\right)$, and $3\left(4 d^{10} 5 s^{2} 5 p^{2}\right)$, respectively. We present in this manuscript a detailed study of the structural, electronic, and magnetic properties of antiferromagnetic $\mathrm{Mn}_{2} \mathrm{PtSn}$ Heusler compounds, in the both phase structures. The volume and the $c / a$ ratio optimization has been performed and fitted by the Murnaghan equation of state [15] to obtain the ground state lattice parameters. The equilibrium lattice constant $a$, the $c / a$ ratio, bulk modulus $B$ and its pressure derivatives $B^{\prime}$ for both the cubic and tetragonal phase have been computed and are listed in Table I for the $\mathrm{Mn}_{2} \mathrm{PtSn}$ compounds. We conclude that our results are in excellent agreement with that obtained from other studies. As an example, Wollmann [16] proved that the tetragonal structure is more stable for this material because the difference between the minimum energy for the two structures is negative $(\Delta E<0)$, while on the other hand the cubic structure is unstable.

\subsection{Mechanical properties and Debye temperature}

In the first framework, we have calculated in this part the elastic constants described by three constants for cubic phase that is $C_{11}, C_{12}$, and $C_{44}$, and six independent constants for tetragonal symmetry, namely $C_{11}$, $C_{12}, C_{13}, C_{33}, C_{44}$, and $C_{66}$. These constants are very important for describing the different properties such as stability, stiffness, hardness of materials because these constants determine the response of the crystal to an external stress characterized by the modulus of elasticity as a bulk modulus, Young modulus, shear modulus, and Poisson ratio $[17,18]$.
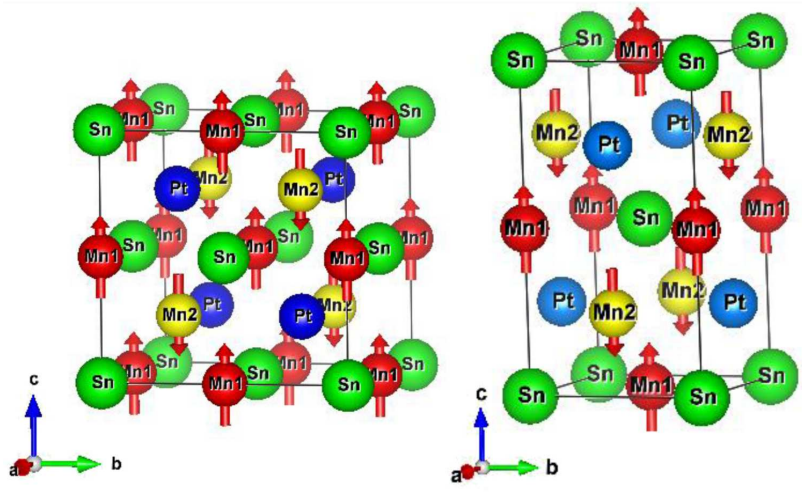

Fig. 1. Crystal and magnetic structure of $\mathrm{Mn}_{2} \mathrm{PtSn}$, for the two structures: inverse cubic structure $(F \overline{4} 3 \mathrm{~m})$ and the inverse tetragonal structure $I \overline{4} m 2$, with Mn1 (red spheres) sitting in the Mn-Sn plane and Mn2 (yellow spheres) sitting in the $\mathrm{Mn}-\mathrm{Pt}$ planes. The $\mathrm{Sn}$ atoms are shown as green spheres. The arrows represent the direction of the magnetic moment at each atom.

TABLE I

The lattice parameters $a, c / a$, bulk modulus $B(\mathrm{GPa})$, relative volume change $C_{t / c}$ and energy differences $\Delta E$ between the cubic and tetragonal phases, respectively, for $\mathrm{Mn}_{2} \mathrm{PtSn}$ compared with other experimental and theoretical results

\begin{tabular}{c|c|c|c|c|c|c}
\hline \hline Phase & $a[\AA]$ & $c / a$ & $B[\mathrm{GPa}]$ & $B^{\prime}$ & $C_{t / c}$ & $\Delta E[\mathrm{Ry}]$ \\
\hline \multirow{2}{*}{ cubic } & 6.3839 & 1.00 & 119.97 & 4.31 & & \\
& $6.39^{a}$ & & & & & \\
\hline \multirow{5}{*}{ tetragonal } & 4.1421 & 1.825 & 117.13 & 4.84 & -0.328 & -0.11617 \\
& $4.509^{b}$ & $1.3477^{b}$ & & & $-0.35^{a}$ & \\
& $4.512^{c}$ & $1.3484^{c}$ & & & & \\
& $4.201^{d}$ & $1.32^{d}$ & & & & \\
& $4.15^{a}$ & $1.81^{a}$ & & & & \\
\hline
\end{tabular}

In order to compute polycrystalline elastic moduli, we apply the Voigt-Reuss-Hill approximation [19]. In this approach, the actual effective modulus for a polycrystalline system is approximated by the arithmetic mean of the two well-known bounds for monocrystals according to Voigt [20], Reuss, and Agnew [21].

The polycrystalline mechanical properties calculated here include the bulk modulus $B$, shear modulus $G$, Young's modulus E, Poisson's ratio $\sigma$ and the Debye temperature $\theta_{\mathrm{D}}[22,23]$. The mathematical formulation is provided in the following equations:

$$
\begin{aligned}
& B_{\mathrm{V}}=\frac{1}{3}\left(C_{11}+2 C_{12}\right), \quad G_{\mathrm{V}}=\frac{C_{11}-C_{12}+3 C_{44}}{5}, \\
& G_{\mathrm{R}}=\frac{5\left(C_{11}-C_{12}\right) C_{44}}{4 C_{44}+3\left(C_{11}-C_{12}\right)}, \quad B_{\mathrm{V}}=G_{\mathrm{R}} .
\end{aligned}
$$

For cubic solid and for a tetragonal solid

$$
\begin{aligned}
B_{\mathrm{V}} & =\frac{2}{9}\left(C_{11}+C_{12}+2 C_{13}+\frac{C_{33}}{2}\right), \\
G_{\mathrm{V}} & =\frac{12 C_{44}+12 C+C_{11}+C_{12}+2 C_{33}-4 C_{13}}{30},
\end{aligned}
$$




$$
\begin{aligned}
G_{\mathrm{R}} & =\frac{5\left(C_{11}-C_{12}\right) C_{44}}{4 C_{44}+3\left(C_{11}-C_{12}\right)}, \\
B_{\mathrm{R}} & =\frac{C_{33}\left(C_{11}+C_{12}\right)-2 C_{13}^{2}}{C_{11}+C_{12}+2 C_{33}-4 C_{13}} .
\end{aligned}
$$

Hill has estimated the average bulk and shear moduli from relationships

$$
B=1 / 2\left(B_{\mathrm{V}}+B_{\mathrm{R}}\right), \quad G=1 / 2\left(G_{\mathrm{V}}+G_{\mathrm{R}}\right) .
$$

In the above equations, the subscript $V$ denotes the Voigt bound and $R$ denotes the Reuss bound, The Young modulus $E$ and Poisson's ratio $\sigma$ are connected to $B$ and $G$ by relations

$$
E=\frac{9 B G}{3 B+G}, \quad \sigma=\frac{3 B-2 G}{6 B+2 G}
$$

The Debye temperature $\theta_{\mathrm{D}}$ is an important fundamental parameter and is closely related to many physical properties of solids such as the specific heat and melting temperature. One of the standard methods to calculate $\theta_{\mathrm{D}}$ is from elastic constants data, since it may be estimated from the average sound velocity $v_{m}$, using the following equations [24]:

$$
\begin{aligned}
& \theta_{\mathrm{D}}=\frac{h}{k_{\mathrm{B}}}\left(\frac{3 n}{4 \pi V_{a}}\right)^{-\frac{1}{3}} v_{m}, \quad v_{m}=\left[\frac{1}{3}\left(\frac{2}{v_{t}^{3}}+\frac{1}{v_{l}^{3}}\right)\right]^{-\frac{1}{3}}, \\
& v_{l}=\left(\frac{3 B+4 G}{3 \rho}\right)^{0.5}, \quad v_{t}=\left(\frac{G}{\rho}\right)^{0.5} .
\end{aligned}
$$

Here $v_{m}$ is the average sound velocity, $h$ is Planck's constant, $k_{\mathrm{B}}$ is Boltzmann's constant, $V_{a}$ is atomic volume, $v_{l}$ and $v_{t}$ are the longitudinal and transverse sound velocity, respectively. The calculated values of longitudinal, transverse, and average sound velocity $\left(v_{l}, v_{t}\right.$, and $v_{m}$ in $\mathrm{m} / \mathrm{s}$ ) as well as the Debye and melting temperature for both the cubic and tetragonal phases for $\mathrm{Mn}_{2} \mathrm{PtSn}$ are listed in Table II.

TABLE II

The longitudinal, transverse, and average sound velocity $\left(\vartheta_{l}, \vartheta_{t}\right.$ and $\vartheta_{m}$ in $\left.\mathrm{m} / \mathrm{s}\right)$ as well as the Debye $\theta_{\mathrm{D}}$ for $\mathrm{Mn}_{2} \mathrm{PtSn}$ compound

\begin{tabular}{l|c|c|c|c|c}
\hline \hline & $\vartheta_{l}$ & $\vartheta_{t}$ & $\vartheta_{m}$ & $\theta_{\mathrm{D}}$ & Melt. temp. \\
\hline$F \overline{4} 3 m$ & 8830.03 & 7098.6 & 7523.82 & 884.17 & $944.013 \mp 300$ \\
$I \overline{4} m 2$ & 4022.44 & 1972.72 & 2215.48 & 260.59 & $1166.08 \mp 300$
\end{tabular}

For cubic crystal structures, the necessary conditions for mechanical stability are given by

$$
\left\{\begin{array}{l}
C_{11}-C_{12}>0 \\
C_{11}>0, C_{44}>0 \\
C_{11}+2 C_{12}>0 \\
C_{12}<B<C_{11}
\end{array}\right.
$$

The stability criteria for a tetragonal crystal [25] are

$$
\left\{\begin{array}{l}
C_{11}>0, \quad C_{33}>0, \quad C_{44}>0, \quad C_{66}>0 \\
C_{11}-C_{12}>0 \\
C_{11}+C_{33}-2 C_{13}>0 \\
2\left(C_{11}+C_{12}\right)+C_{33}+4 C_{13}>0
\end{array}\right.
$$

Table III shows the numerical values of our computation of all the elastic constants for both the structures. These values for cubic structure satisfy all the stability criteria, except the condition $C_{11}-C_{12}>0$. Thus we have concluded that the $F \overline{4} 3 m \mathrm{Mn}_{2} \mathrm{PtSn}$ is mechanically unstable.

TABLE III

Calculated elastic constants $C_{i j}$ (in GPa), elastic modulus $(G$ and $E$ ) (in GPa), Poisson' ratio $(\nu), B / G$ ratio, shear anisotropic factor $(A)$ for the two different shear planes $\left(A_{1}\right.$ and $\left.A_{2}\right)$ for both the cubic and tetragonal phases for $\mathrm{Mn}_{2} \mathrm{PtSn}$ compound

\begin{tabular}{c|c|c}
\hline \hline Phase & Cubic & Tetragonal \\
\hline$C_{11}$ & 66.16 & 185.18 \\
$C_{12}$ & 141.86 & 56.66 \\
$C_{13}$ & - & 106.58 \\
$C_{33}$ & - & 171.019 \\
$C_{44}$ & 52.17 & 57.26 \\
$C_{66}$ & - & 24.55 \\
$G[\mathrm{GPa}]$ & 90.03 & 42.21 \\
$E[\mathrm{GPa}]$ & 216.04 & 113.26 \\
$A$ & -1.899 & $A_{1}=1.6$, \\
& & $A_{2}=0.382$ \\
$\nu$ & -0.413 & 0.341
\end{tabular}

Only the $I \overline{4} m 2\left(\mathrm{Mn}_{2} \mathrm{PtSn}\right)$ phase was found to be mechanically stable i.e. its elastic constants obey the conditions described in relation (7).

The different elastic constants of cubic and tetragonal $\mathrm{Mn}_{2} \mathrm{PtSn}$ at $0 \mathrm{GPa}$ and $0 \mathrm{~K}$ are listed in Table III. There is currently no experimental measurement of elastic constants for us to compare.

In the present calculations for tetragonal structure $C_{11}>C_{33}$, which exhibited that the bonding strength along the axis [100] and [010] direction is stronger than that of the bonding along the axis [001] direction. Moreover, $C_{66}>C_{44}$ predicts that the shear in axis and plane [100] (010) is easier than shear in the axis and plane [100] (001), respectively.

The ratio $B / G$ indicates the ductility or brittleness of the materials, the factor that indicates the resistance to change of volume by the applied pressure which is the bulk module. On the other hand, the resistance to plastic deformation is represented by the shear modulus $G$.

The $B / G$ value for the tetragonal structure is 2.77 which proves that the $\mathrm{Mn}_{2} \mathrm{PtSn}$ material behaves ductile.

Elastic or thermal anisotropy is responsible for the microcracks induced in alloys. To measure the degree of anisotropy in the bond between the atoms in different planes it is necessary to calculate the anisotropic shear factors. The anisotropic shear factors along shear planes $\{100\}$ and $\{001\}$ are defined as follows [26]:

$$
\left\{\begin{array}{l}
A_{1}=A_{\{100\}}=\frac{4 C_{44}}{C_{11}+C_{33}-2 C_{13}} \\
A_{2}=A_{\{001\}}=\frac{2 C_{66}}{C_{11}-C_{12}}
\end{array}\right.
$$



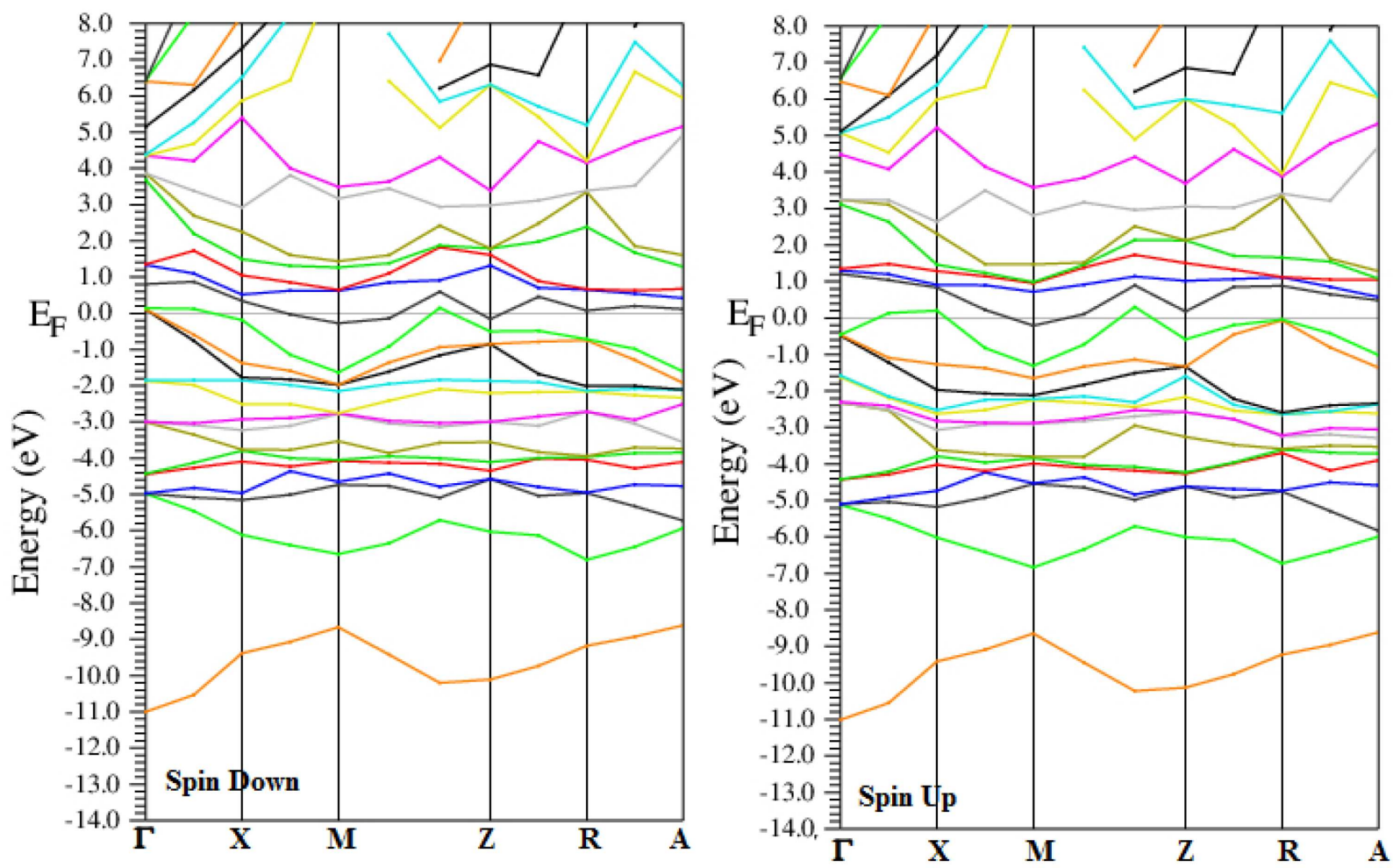

Fig. 2. Calculated the spin up and down band structures for tetragonal $(I \overline{4} m 2) \mathrm{Mn}_{2} \mathrm{PtSn}$ along the high-symmetry axes of the first Brillouin zone.

The measure of the degree of elastic anisotropy of the crystal is given by the deviation of $A_{1}$ and $A_{2}$ to a one. In the case of an isotropic crystal the factors $A_{1}, A_{2}$ must be equal to one. Our results indicate that the elastic anisotropy is not unity $(A \neq 1)$ which means that the Young modulus $E(r)$ exhibits a non-spherical distribution, and the crystal would not be stable in the cubic structure due to the Born-Huang criteria [27]. We note that there is no experimental data to verify our results.

\subsection{Electronic and magnetic structure}

The existence of a high state density peaks (DOS) near $E_{\mathrm{F}}$ indicates the structural instability of the cubic phase, which is the van Hove singularity [28]. Several attempts have been found in literature to explain the instability of the cubic phase using the Teller effect [29, 30], anomalous phonon vibrations [31, 32], and the Fermi-surface nesting [33].

For the Heusler compound $\mathrm{Mn}_{2} \mathrm{PtSn}$, the coordinated Mn atoms of octahedra generally have the $d^{4}$ configuration of the $\mathrm{Mn}_{2}^{+3}$ ions and are therefore very sensitive to the Jahn-Teller distortions. If the corresponding degenerated $e_{g}$ states are found at $E_{\mathrm{F}}$ for $c / a=1$, the local minimum of the total energy will be shifted towards $c / a=1.8$.

In Fig. 2 we present the band structure of $\mathrm{Mn}_{2} \mathrm{PtSn}$ for the two spin directions (up and down). It is obvious from the result that our compound has a metallic overlap at the Fermi level because of the approach we used.
Since, we find in our materials the electrons of $3 d$ and $5 d$ transition metals which are strongly correlated, it is necessary to provide another approach to solve this problem, a work that we hope to do in the future.

We try to give an explanation on the instability of the cubic phase based on the DOS study. For the cubic phase, the partial PDOS is shown in Fig. 3a.

The cubic phase is unstable as we see clearly the redistribution of spectral density above the Fermi energy, such that a high DOS near $E_{\mathrm{F}}$ is moved down by the overture up of a "valley" near $E_{\mathrm{F}}$ in the tetragonal phase. It is seen that the redistribution occurs particularly in $e_{g}$ states with considerable overlap of the $t_{2 g}$ states in the majority channel. The $\operatorname{Mn}(4 \mathrm{~b}) d$-states are separated because of the strong crystal field splitting. We note that the occupied $e_{g}$ states are situating in an interval between -3.25 and $-1.65 \mathrm{eV}$. On the other hand, the $t_{2 g}$ states in PDOS is found between $-1.25 \mathrm{eV}$ and the Fermi level $E_{\mathrm{F}}$. Although then, separation of the occupied and empty states prohibits the strong exchange split of the $\operatorname{Mn}(4 \mathrm{~b}) d$-states, in contrast to the $d$-states of $\operatorname{Pt}(4 \mathrm{c})$ and $\mathrm{Mn}(4 \mathrm{~d})$, which are found to be more extensively dispersed, even if the majority and minority states are separated owing to exchange splitting.

The PDOS of the cubic phase are comparable to the PDOS of the tetragonally distorted systems, Therefore, we note that the resulting PDOS are widely scattered and significantly less structured. 

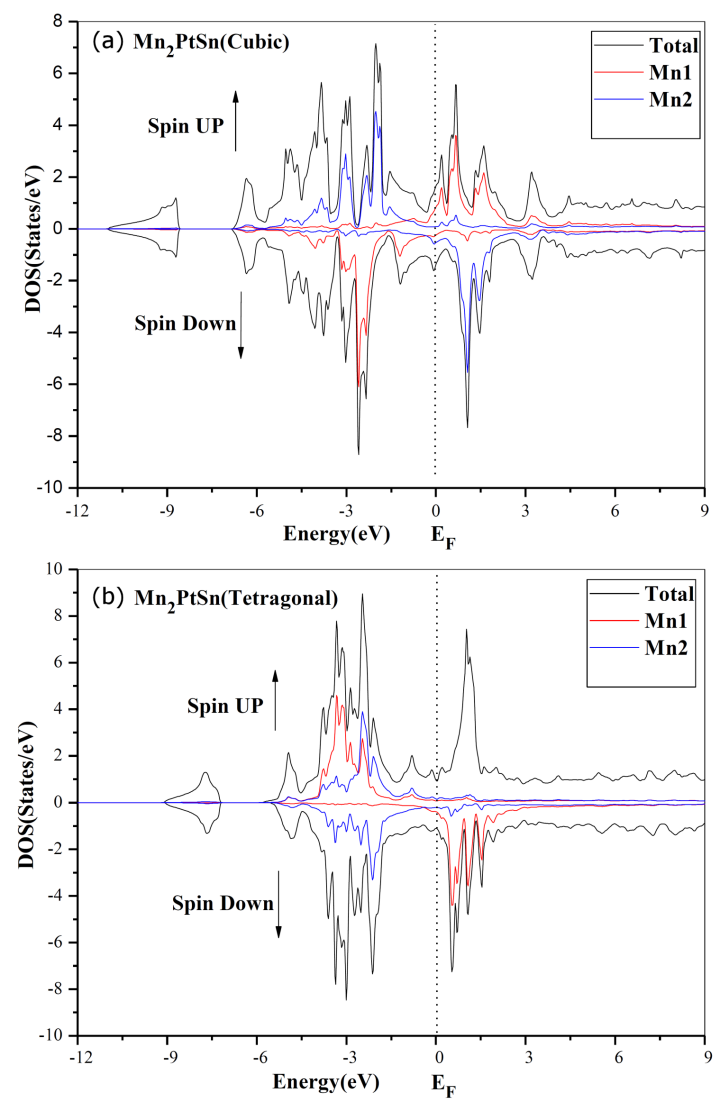

Fig. 3. Density of states of (a) cubic $(F \overline{4} 3 m)$ and (b) tetragonal $(I \overline{4} m 2) \mathrm{Mn}_{2} \mathrm{PtSn}$.

In Fig. 2b we present the total and partial DOS for tetragonal phase. It can be found that the general shapes of the cubic and tetragonal DOS are quite similar, with disappearance of the peaks in the tetragonal DOS at $3 \mathrm{eV}$ in majority and minority states.

The amount of DOS at $E_{\mathrm{F}}$ is decreased after the tetragonal distortion which increases the spin polarization for this phase. The same mechanism has been observed for $\mathrm{Mn}_{2} \mathrm{PtIn}$ [34]. In Fig. 3 we clearly see a great contribution for the majority spins due to the $d$ states of Mn1 in the conduction band and it disappears during the tetragonal distortion. The cubic phase is highly symmetrical than the tetragonal phase, which influences the distribution of the DOS around the Fermi level and even on the distribution of the $3 d$ states for the two Mn.

In $\mathrm{Mn}_{2}$-based Heusler family compounds (cubic or tetragonal) we can find the antiparallel alignment of the magnetic moments in both of the different Mn sublattices. Although, not all $\mathrm{Mn}_{2} \mathrm{YZ}$ accept noncollinearity of magnetic moment. The typical collinear ferrimagnetic state is due to a very significant exchange coupling between the nearest Mn1 (2b) and Mn2 (2d) atoms, which is characterized by a high exchange constant.

The collinear ferrimagnetic order cannot explain the measured saturated magnetization in the tetragonal $\mathrm{Mn}_{2} \mathrm{RhSn}, \mathrm{Mn}_{2} \mathrm{PtIn}$, and $\mathrm{Mn}_{2} \mathrm{IrIn}$. For $\mathrm{Mn}_{2} \mathrm{PtSn}$ we find that the collinear order can be agitated by the next important interaction $j$ couplings between the $\mathrm{Mn}(2 \mathrm{~d})-\mathrm{Pt}$ planes.

This interaction is antiparallel due to its indirect interaction origin realized through the $\mathrm{Sn}$ atom. The exchange constant $j$ has a tendency to pivot the antiparallel moments to each other of the closest $\mathrm{Mn}(2 \mathrm{~d})-\mathrm{Pt}$ planes.

In the $\mathrm{Mn}(2 \mathrm{~d})-\mathrm{Pt}$ plane, the $\mathrm{Mn}$ moment indicates the value of $3.344 \mu_{\mathrm{B}}$ which couples antiferromagnetically to the Mn moments in the Mn-Mn plane, which have a low total moment of $0.037 \mu_{\mathrm{B}}$ for tetragonal structure. The magnetic moment value of $\mathrm{Mn}_{2} \mathrm{PtSn}$ for the antiferromagnetic order is $3.344 \mu_{\mathrm{B}} / \mathrm{Mn}$ which is larger than the experimental saturation magnetic moment value $1.9 \mu_{\mathrm{B}} / \mathrm{Mn}[35]$ and is in good agreement with the value $3.3 \mu_{\mathrm{B}} / \mathrm{Mn}$ predicted theoretically [16].

In Table IV we presented the total magnetic moment as the combination of the moments at the $\mathrm{Mn}, \mathrm{Pt}$, and Sn sites with comparisons a literature data. This big difference between the calculated and experimental moment value is due to the real spin structure in the inverse tetragonal $\mathrm{Mn}_{2} \mathrm{PtSn}$. This deficit may be different from the one assumed in the theoretical calculation where the collinear situation of the magnetic moment is not applicable to this compound. We note that $\mathrm{Mn}_{2} \mathrm{PtSn}$ follow the isostructural model $\mathrm{Mn}_{2} R h S n$ which requires that the spin structure to be non-collinear [36].

TABLE IV

Calculated total and local magnetic moment (in Bohr magnetons $\mu_{\mathrm{B}}$ ) for both the cubic and tetragonal phases in the ferromagnetic and antiferromagnetic order for $\mathrm{Mn}_{2} \mathrm{PtSn}$.

\begin{tabular}{|c|c|c|c|c|c|c|}
\hline \multicolumn{2}{|c|}{ Phase } & $M_{\text {tot }}$ & $M_{\mathrm{Mn} 1}$ & $M_{\mathrm{Mn} 2}$ & $M_{\mathrm{Pt}}$ & $M_{\mathrm{Sn}}$ \\
\hline \multirow[b]{2}{*}{$\frac{.}{3}$} & FM & 7.05 & 2.985 & 3.342 & 0.282 & -0.034 \\
\hline & AFM & $\begin{array}{l}0.185 \\
0.19^{a}\end{array}$ & -3.202 & 3.427 & 0.0406 & 0.016 \\
\hline \multirow{2}{*}{ 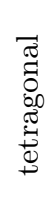 } & FM & $\begin{array}{c}6.99 \\
6.66^{b}\end{array}$ & 3.384 & 3.065 & 0.179 & -0.06 \\
\hline & AFM & $\begin{array}{c}0.028 \\
-0.02^{a}\end{array}$ & -3.307 & $\begin{array}{c}3.344 \\
3.3^{b} \\
1.9^{c}\end{array}$ & 0.001 & 0.00007 \\
\hline
\end{tabular}

${ }^{a}$ Ref. [16], ${ }^{b}$ Ref. [4], ${ }^{c}$ Ref. [35]

The Heusler tetragonal compound is a promising new generation of hard magnets. $\mathrm{Mn}_{2} \mathrm{PtSn}$ possesses a very high magnetocrystalline anisotropy energy (MAE) because it has a number of electrons with a valence of $N_{V}=28$ which is directly related to $\Delta E$, the energy difference between the van Hove singularity and $E_{\mathrm{F}}$, and a particularly high conductivity importantly causing a weak crystallographic symmetry. The highest magnetocrystalline anisotropy (MCA) is 3.04 for $\mathrm{Mn}_{2} \mathrm{PtSn}$ with low $T_{\mathrm{C}}=374 \mathrm{~K}$ [4], which becomes a disadvantage. The materials that have a high MCA are also good candidates for STT applications. It remains to settle the $T_{\mathrm{C}}$ for $\mathrm{Mn}_{2} \mathrm{PtSn}$ either by doping or substitution. 
The distortion tetragonal can induce a very high MAE of up to $5 \mathrm{MJ} \mathrm{m}^{-3}$ for the Heusler compounds containing $\mathrm{Pt}$, comparable to the best known transition metal magnet L10-FePt, and of up to $1 \mathrm{MJ} \mathrm{m}^{-3}$ for the Co rich but $\mathrm{Pt}$ free Heusler compounds [37]. In the case of $\mathrm{Mn}_{2} \mathrm{PtSn}$ where a distortion occurs the volume change is found to be very small nearly $0.19 \%$.

The spin polarization is complete or almost complete, $P\left(E_{\mathrm{F}}\right) \approx 100 \%$, and it has been observed in cubic $\mathrm{Mn}_{2}-$ based Heusler compounds [38, 39]. From the density of state, we can calculate the degree of spin polarization at the Fermi level by the relation

$$
P=\frac{N\left(E_{\mathrm{F}}\right) \uparrow-N\left(E_{\mathrm{F}}\right) \downarrow}{N\left(E_{\mathrm{F}}\right) \uparrow+N\left(E_{\mathrm{F}}\right) \downarrow},
$$

$P$ disappears below the magnetic transition temperature in paramagnetic or in anti-ferromagnetic materials. Because of the approach used, we found a value of $P$ smaller than the value cited in literature because of the absence of gap at the Fermi level for the minotary spin.

\subsection{Exchange coupling and Curie temperature}

Using the Liechtenstein formula [40] to calculate the interactions of exchange, according to the Heisenberg model the total exchange is $J_{\text {tot }}=J_{\text {indirect }}+J_{\text {direct }}$. The behavior of exchange interactions in Mn2-based Heusler compounds is complex and short ranged. The conduction $s p$ electrons play a critical role described by Anderson's $s d$ model. We can express $J_{\text {indirect }}$ in terms of interaction Ruderman-Kittel-Kasuya-Yosida (RKKY) [41-43].

The Curie temperature $T_{\mathrm{C}}$ and the exchange interaction $J_{i j}$ are attached by the following relation [31]:

$$
T_{\mathrm{C}} k_{\mathrm{B}}=\frac{2}{3} \sum_{i \neq j} J_{i j} .
$$

Here $k_{\mathrm{B}}$ is the Boltzmann constant $\left(k_{\mathrm{B}}=1.38064852 \times\right.$ $\left.10^{-23} \mathrm{~J} / \mathrm{K}\right)$ and $J_{i j}$ is the exchange interaction defined by the relation:

$$
J_{i j}=\frac{E_{\mathrm{FM}}-E_{\mathrm{AFM}}}{2} .
$$

Here $E_{\mathrm{FM}}$ is the total energy of the ferromagnetic state (parallel spins), $E_{\mathrm{AFM}}$ is that for the antiferromagnetic state (anti-parallel adjacent spins).

We summarize, in Table V, the Curie temperature and the spin polarization for both the cubic and tetragonal phases for the $\mathrm{Mn}_{2} \mathrm{PtSn}$ compared with the available data.

\section{TABLE V}

The calculated spin polarization at the Fermi level $P_{c}, P_{t}$, the Curie temperature in kelvins of tetragonal $T_{\mathrm{C}, t}$ and cubic $T_{\mathrm{C}, c}$ for $\mathrm{Mn}_{2} \mathrm{PtSn}$ compound. The changes due to the tetragonal transformation are listed as $\Delta T_{\mathrm{C}, t-c}$.

\begin{tabular}{c|c|c|c|c}
\hline \hline$P_{c}$ & $P_{t}$ & $T_{\mathrm{C}, c}$ & $T_{\mathrm{C}, t}$ & $\Delta T_{\mathrm{C}, t-c}$ \\
\hline $8.93 \%$ & $24.74 \%$ & $737.27 \mathrm{~K}$ & $215.01 \mathrm{~K}$ & $-521.74 \mathrm{~K}$ \\
& $91 \%^{a}$ & & $374 \mathrm{~K}^{a}$ & \\
\hline
\end{tabular}

${ }^{a}$ Ref. [4, 5]
As mentioned in Table $\mathrm{V}$, one of the main issues associated with $\mathrm{Mn}_{2} \mathrm{PtSn}$ is its low Curie temperature (the calculated value $\left.T_{C}=215 \mathrm{~K}\right)$. According to the work of Huh et al. (see Fig. 3b of Ref. [35]), the Curie temperature can be increased by increase of the lattice parameters $a$ and decrease of the ratio $c / a$.

We have found that the $T_{C}$ can be substantially increased by substituting the another transition element, for example, Co for a fraction of $\mathrm{Pt}$ atoms in $\mathrm{Mn}_{2} \mathrm{PtSn}$.

\section{Conclusion}

We have studied the mechanical, electronic, and magnetic properties for both phases of $\mathrm{Mn}_{2} \mathrm{PtSn}$ compound using the full-potential linearized augmented plane wave. The elastic constants are calculated, yielding the related mechanical properties including bulk modulus $B$, shear modulus $G$, Young's modulus $E$, Poisson's ratio $\nu$, and the Debye temperature. We confirmed employing the stability criteria that $\mathrm{Mn}_{2} \mathrm{PtSn}$ favors the tetragonal phase. We predict that the tetragonal phase of $\mathrm{Mn}_{2} \mathrm{PtSn}$ has a Curie temperature $\left(T_{\mathrm{C}}\right)$ below the ambient temperature which becomes an obstacle for technological applications. The magnetic properties are also investigated for the ferromagnetic and antiferromagnetic order, and we note that the total magnetic moment of the tetragonal phase is a little larger than that of the cubic phase. This compound at this phase may be promising candidate for spin-transfer-torque applications as a consequence. Also, it is important to indicate that the degree of spin polarization is smaller than the experimental value which obliges us to adjust it and determine if this material is useful for STT-based devices.

\section{References}

[1] Y. Huh, P. Kharel, V.R. Shah, E. Krage, R. Skomski, J.E. Shield, D.J. Sellmyer, IEEE Trans. Magn. 49, 3277 (2013).

[2] H. Kurt, K. Rode, M. Venkatesan, P. Stamenov, J.M.D. Coey, Phys. Rev. B 83, 020405 (2011).

[3] C. Felser, V. Alizani, J. Winterlik, S. Chadov, A.K. Nayak, IEEE Trans. Magn. 49, 682 (2013).

[4] J. Kubler, C. Felser, Phys. Rev. B 85, 012405 (2012).

[5] J. Winterlik, A. Gupta, V. Alijani, T. Gasi, K. Filsinger, B. Balke, G.H. Fecher, C.A. Jenkins, F. Casper, J. Kübler, G.D. Liu, L. Gao, S.S.P. Parkin, C. Felser, Adv. Mater. 24, 6283 (2012).

[6] D. Zhang, B. Yan, S.C. Wu, J. Kübler, G. Kreiner, S.S.P. Parkin, C. Felser, J. Phys. Condens. Matter 25, 206006 (2013).

[7] A. Nelson, Y. Huh, P. Kharel, V.R. Shah, R. Skomski, D.J. Sellmyer, J. Appl. Phys. 115, 17A923 (2014).

[8] J.C. Slater, Adv. Quant. Chem. 1, 5564 (1964).

[9] P. Blaha, K. Schwarz, G.K.H. Madsen, D. Kvasnicka, J. Luitz, WIEN2K, An Augmented Plane Wave Local Orbitals Program for Calculating Crystal Properties, 2001. 
[10] P. Blaha, K. Schwarz, P. Sorantin, S.B. Tricky, Comput. Phys. Commun. 59, 399 (1990).

[11] K. Schwarz, P. Blaha, G.K.H. Madsen, Comput. Phys. Commun. 147, 71 (2002).

[12] J.P. Perdew, S. Burke, M. Ernzerhof, Phys. Rev. Lett. 77, 3865 (1996).

[13] J.P. Perdew, S. Burke, Y. Wang, Phys. Rev. B 54, 16533 (1996).

[14] H.J. Monkhorst, J.D. Pack, Phys. Rev. B 13, 5188 (1976).

[15] F.D. Murnaghan, Proc. Natl. Acad. Sci. USA 30, 244247 (1944).

[16] L. Wollmann, S. Chadov, J. Kübler, C. Felser, Phys. Rev. B 92, 064417 (2015).

[17] M. Jamal, N.K. Sarvestani, A. Yazdani, A.H. Reshak, RSC Adv. 4, 57903 (2014).

[18] A.H. Reshak, M. Jamal, Int. J. Electrochem. Sci. 8, 12252 (2013).

[19] R. Hill, Proc. Phys. Soc. 65, 349 (1952).

[20] W. Voigt, Lehrbuch der Kristallphysik, Teubner, Leipzig 1928.

[21] A. Reuss, A. Agnew, Math. Mech. 9, 49 (1929).

[22] O.L. Anderson, J. Phys. Chem. Solids 24, 909 (1963).

[23] E. Schreiber, O.L. Anderson, N. Soga, Elastic Constants and Their Measurement, McGraw-Hill, New York 1973.

[24] D.C. Wallace, Thermodynamics of Crystals, Wiley, New York 1972, Ch. 1.

[25] G.V. Sinko, N.A. Smirnow, J. Phys. Condens. Matter 14, 6989 (2002).

[26] M. Born, K. Huang, Dynamical Theory of Crystal Lattices, Clarendon Press, Oxford 1956.

[27] L.V. Hove, Phys. Rev. 89, 1189 (1953).

[28] J. Winterlik, B. Balke, G.H. Fecher, C. Felser, M.C.M. Alves, F. Bernardi, J. Morais, Phys. Rev. $B$ 77, 054406 (2008).
[29] T. Gasi, A.K. Nayak, J. Winterlik, V. Ksenofontov, P. Adler, M. Nicklas, C. Felser, Appl. Phys. Lett. 102, (2013).

[30] A.T. Zayak, P. Entel, K.M. Rabe, W.A. Adeagbo, M. Acet, Phys. Rev. B 72, 054113 (2005).

[31] P. Entel, V.D. Buchelnikov, V. Khovailo, A.T. Zayak, W.A. Adeagbo, M.E. Gruner, H.C. Herper, E.F. Wassermann, J. Phys. D Appl. Phys. 39, 865 (2006).

[32] S.R. Barman, S. Banik, A. Shukla, C. Kamal, A. Chakrabarti, Europhys. Lett. 80, 57002 (2007).

[33] Hongzhi Luo, Pengzhong Jia, Guodong Liu, Fanbin Meng, Heyan Liu, Enke Liu, Wenhong Wang, Guangheng Wu, Solid State Communications 170, 44 (2013).

[34] Y. Huh, P. Kharel, A. Nelson, V.R. Shah, J. Pereiro, P. Manchanda, A. Kashyap, R. Skomski, D.J. Sellmyer, J. Phys. Condens. Matter 27, 076002 (2015).

[35] O. Meshcheriakova, S. Chadov, A.K. Nayak, U.K. Rößler, J. Kübler, G. André, A.A. Tsirlin, J. Kiss, S. Hausdorf, A. Kalache, W. Schnelle, M. Nicklas, C. Felser, Phys. Rev. Lett. 113, 087203 (2014).

[36] Y.I. Matsushita, G. Madjarova, J.K. Dewhurst, S. Shallcross, C. Felser, S. Sharma, E.K.U. Gross, J. Phys. D Appl. Phys. 50, 095002 (2017).

[37] J.K. Ubler, A.R. Williams, C.B. Sommers, Phys. Rev. $B$ 28, 1745 (1983).

[38] R.A. de Groot, F.M. Mueller, P.G. van Engen, K.H.J. Buschow, Phys. Rev. Lett. 50, 2024 (1983).

[39] A.I. Liechtenstein, M.I. Katsnelson, P.V. Antropov, A.V. Gubanov, J. Magn, Mater. 67, 65 (1987).

[40] M.A. Ruderman, C. Kittel, Phys. Rev. 96, 99 (1954).

[41] T. Kasuya, Prog. Theor. Phys. 16, 45 (1956).

[42] K. Yosida, Phys. Rev. 106, 893 (1957). 